\title{
Entropy diminishing finite volume approximation of a cross-diffusion system
}

\author{
Clément Cancès and Benoît Gaudeul
}

\begin{abstract}
We propose a two-point flux approximation finite volume scheme for the approximation of the solutions of a entropy dissipative cross-diffusion system. The scheme is shown to preserve several key properties of the continuous system, among which positivity and decay of the entropy. Numerical experiments illustrate the behaviour of our scheme.
\end{abstract}

Key words: Cross-diffusion system, Entropy dissipative scheme

MSC (2010): 65M08, 65M12, 35K51

\section{Finite Volume approximation of a cross diffusion system}

The model addressed in this paper is a toy model for the evolution of a material [1] which can be derived thanks to a jump process following the program of [3]. We are interested in the evolution of the composition of the material, which is described by the concentrations $\mathbf{c}=\left(c_{1}, \ldots, c_{I}\right)$ of $I$ different species. The material is represented by an open, connected, bounded, and polyhedral subset $\Omega$ of $\mathbb{R}^{d}$, and the evolution of its composition is prescribed by the following system of partial differential equations. The mass conservation of each species writes for all $i \in\{1, \ldots, I\}$

$$
\partial_{t} c_{i}+\nabla \cdot \mathbf{J}_{i}=0 \quad \text { in } \mathbb{R}_{+} \times \Omega, \quad \text { with } \quad \mathbf{J}_{i}=\sum_{j \neq i} \kappa_{i j}\left(c_{i} \nabla c_{j}-c_{j} \nabla c_{i}\right) .
$$

\footnotetext{
Clément Cancès

Inria, Univ. Lille, CNRS, UMR 8524 - Laboratoire Paul Painlevé, F-59000 Lille, France

e-mail: clement.cances@inria.fr

Benoît Gaudeul

Univ. Lille, CNRS, UMR 8524, Inria - Laboratoire Paul Painlevé, F-59000 Lille, France

e-mail: benoit.gaudeul@univ-lille.fr
} 
The coefficients $\kappa_{i j}$ are such that $\kappa_{i j}=\kappa_{j i} \geq 0$. The system is complemented with no-flux boundary conditions $\mathbf{J}_{i} \cdot \mathbf{n}=0$ on $\partial \Omega$, and an initial condition $\mathbf{c}^{0}=$ $\left(c_{1}^{0}, \ldots, c_{I}^{0}\right)$ which satisfies $\langle\mathbf{c}, \mathbf{1}\rangle=\sum_{i=1}^{I} c_{i}^{0}=1$ in $\Omega$.

This continuous problem has some key-properties that one wants to preserve after discretisation. First, the total mass of each specie is conserved, i.e, $\int_{\Omega} c_{i}(t)=\int_{\Omega} c_{i}^{0}$ for all $i \in\{1, \ldots, I\}$ and $t \geq 0$. This follows directly from the local conservation property (1) and the no-flux boundary conditions across $\partial \Omega$. Second, the concentrations remains non-negative, i.e., $c_{i}(\mathbf{x}, t) \geq 0$. Third, the expression (1) of $\mathbf{J}_{i}$ and the condition $\kappa_{i j}=\kappa_{j i}$ yield $\sum_{i=1}^{I} \mathbf{J}_{i}=\mathbf{0}$, so that $\sum_{i=1}^{I} c_{i}(\mathbf{x}, t)=1$ for all $(\mathbf{x}, t) \in \Omega \times \mathbb{R}_{+}$. Therefore, $\mathbf{c}(t)$ takes values in the closed convex set

$$
\mathscr{A}=\left\{\mathbf{c} \in L^{1}\left(\Omega ; \mathbb{R}_{+}^{I}\right) \mid\langle\mathbf{c}, \mathbf{1}\rangle=1 \text { a.e. in } \Omega \text { and } \int_{\Omega} c_{i}=\int_{\Omega} c_{i}^{0}\right\} .
$$

Finally, the fluxes rewrite $\mathbf{J}_{i}=-\sum_{j \neq i} \kappa_{i j} c_{i} c_{j} \nabla\left(\log \left(c_{i}\right)-\log \left(c_{j}\right)\right)$. Therefore, multiplying (1) by $\log \left(c_{i}\right)$ and integrating over $\Omega$ leads to

$$
\frac{\mathrm{d}}{\mathrm{d} t} \mathfrak{E}(\mathbf{c})=-\sum_{\{i, j\} \in\{1, \ldots, I\}^{2}} \int_{\Omega} \kappa_{i j} c_{i} c_{j}\left|\nabla\left(\log \left(c_{i}\right)-\log \left(c_{j}\right)\right)\right|^{2} \leq 0,
$$

where the entropy $\mathfrak{E}$ is the convex functional on $\mathscr{A}$ defined by

$$
\mathfrak{E}(\mathbf{c})=\sum_{i=1}^{I} \int_{\Omega} c_{i} \log \left(c_{i}\right)
$$

Then due to the entropy / entropy dissipation relation (2), $t \mapsto \mathfrak{E}(\mathbf{c}(t))$ is nonincreasing, and even decreasing unless $\mathbf{c}$ is constant w.r.t. space.

Under appropriate conditions on the coefficients $\kappa_{i j}$, the existence of weak solutions to the problem can be established thanks to the so-called entropy method [6, 7]. Strong solutions have been recently investigated in [2].

Our goal is to define a scheme that preserves at the discrete level the above properties, i.e. such that the approximate solution belongs to $\mathscr{A}$ for all time and with a discrete counterpart of (2). To this end, we still need to remark that if the coefficients $\kappa_{i j}$ are equal to $\kappa^{\star}>0$, then $\mathbf{J}_{i}=-\kappa^{\star} \nabla c_{i}$, so that (1) reduces to $I$ decoupled heat equations. Therefore, choosing $\kappa^{\star}>0$ and setting $\widetilde{\kappa}_{i j}=\kappa_{i j}-\kappa^{\star}, \mathbf{J}_{i}$ rewrites as

$$
\mathbf{J}_{i}=-\sum_{j \neq i} \widetilde{\kappa}_{i j} c_{i} c_{j} \nabla\left(\log \left(c_{i}\right)-\log \left(c_{j}\right)\right)-\kappa^{\star} \nabla c_{i}, \quad i \in\{1, \ldots, I\} .
$$

Our approach consists in approximating the fluxes $\mathbf{J}_{i}$ under their above form (3). Since it is based on two-point flux approximation (TPFA) finite volumes, it requires the use of a so-called $\Delta$-admissible mesh. Let $\left(\mathscr{T}, \mathscr{E},\left(\mathbf{x}_{K}\right)_{K \in \mathscr{T}}\right)$ be a finite volume mesh of $\Omega$ fulfilling the classical orthogonality condition required for the consistency of TPFA. Since this notion is classical, we remain sloppy here on the definition and refer to [5, Definition 9.1] or to the companion paper [4] for details. Let us just mention that $\mathscr{T}$ denotes the set of the cells, while only internal edges are con- 
sidered in the set $\mathscr{E}$, i.e. $\mathscr{E}=\{\sigma=K \mid L=\partial K \cap \partial L$ for $K, L \in \mathscr{T}\}$. Given $K \in \mathscr{T}$, we denote by $\mathscr{E}_{K}=\{\sigma \in \mathscr{E} \mid \sigma \subset \partial K\}$ and by $m_{K}$ the $d$-dimensional Lebesgue measure of $K$. For $\sigma=K \mid L$, we denote by $m_{\sigma}$ the $(d-1)$-dimensional Lebesgue measure of $\sigma$, by $d_{\sigma}=\left|\mathbf{x}_{K}-\mathbf{x}_{L}\right|$ the distance between the cell centers, and by $a_{\sigma}=\frac{m_{\sigma}}{d_{\sigma}}$ the transmissivity of $\sigma$. For the time discretisation, we allow for non-uniform time steps $\tau_{n}=t^{n}-t^{n-1}, n \geq 1$, with $t^{0}=0$. The initial condition is discretised into

$$
c_{i, K}^{0}=\frac{1}{|K|} \int_{K} c_{i}^{0}, \quad \forall K \in \mathscr{T}, i \in\{1, \ldots, I\} .
$$

In particular, the corresponding piecewise constant reconstruction $\mathbf{c}_{\mathscr{T}}^{0}=\left(c_{i, \mathscr{T}}^{0}\right)_{i}$, defined by $c_{i, \mathscr{T}}^{0}(\mathbf{x})=\sum_{K \in \mathscr{T}} c_{i, K}^{0} \chi_{K}(\mathbf{x})$, belongs to $\mathscr{A}$ provided $\mathbf{c}^{0}$ does. Now, we assume that $\left(c_{i, K}^{n-1}\right)_{i, K}$ is given and is such that the corresponding piecewise constant reconstruction $\mathbf{c}_{\mathscr{T}}^{n-1}$ belongs to $\mathscr{A}$, then we seek $\left(c_{i, K}^{n}\right)_{i, K}$ solution of the following nonlinear system. First, the conservation of mass is locally enforced on each cell $K$ :

$$
\frac{c_{i, K}^{n}-c_{i, K}^{n-1}}{\tau_{n}}|K|+\sum_{\sigma \in \mathscr{E}_{K}} m_{\sigma} J_{i, K \sigma}^{n}=0, \quad \forall K \in \mathscr{T}, i \in\{1, \ldots, I\} .
$$

No flux boundary conditions translate to $J_{i, K \sigma}^{n}=0$ if $\sigma \subset \partial \Omega$. The discretisation of the fluxes $J_{i, K \sigma}^{n} \simeq \mathbf{J}_{i} \cdot \mathbf{n}_{K \sigma}$ across the edge $\sigma=K \mid L$ relies on the expression (3) and writes

$$
J_{i, K \sigma}^{n}=\kappa^{\star} \frac{c_{i, K}^{n}-c_{i, L}^{n}}{d_{\sigma}}+\sum_{j \neq i} \widetilde{\kappa}_{i j}\left(c_{j, \sigma}^{n} \frac{c_{i, K}^{n}-c_{i, L}^{n}}{d_{\sigma}}-c_{i, \sigma}^{n} \frac{c_{j, K}^{n}-c_{j, L}^{n}}{d_{\sigma}}\right)=-J_{i, L \sigma}^{n} .
$$

Finally, the edge concentrations $c_{i, \sigma}^{n}$ are computed from the cell concentrations $c_{i, K}^{n}$ and $c_{i, L}^{n}$ thanks to the continuous formula

$$
c_{i, \sigma}^{n}= \begin{cases}c_{i, K}^{n} & \text { if } c_{i, K}^{n}=c_{i, L}^{n}, \\ \frac{c_{i, K}^{n}-c_{i, L}^{n}}{\log \left(c_{i, K}^{n}\right)-\log \left(c_{i, L}^{n}\right)} & \text { if } c_{i, K}^{n} \neq c_{i, L}^{n}, c_{i, K}^{n}>0, c_{i, L}^{n}>0, \\ 0 & \text { if } \min \left(c_{i, K}^{n}, c_{i, L}^{n} \leq 0 .\right.\end{cases}
$$

The goal of this paper is to show that the scheme (5)-(7) suitably approximates the solutions to (1). This encompasses some mathematical properties of the scheme to be discussed in Section 2 and numerical results presented in Section 3.

Remark 1. Before going further, let us just highlight why the introduction of the positive parameter $\kappa^{\star}$ is important. Assume for simplicity that $I=2$, so that the problem reduces to two uncoupled heat equations on $c_{1}$ and $c_{2}=1-c_{1}$. Assume that the mesh $\mathscr{T}$ is made of two cells $K$ and $L$ separated by the unique edge $\sigma$, and that $c_{1, K}^{0}=1, c_{2, K}^{0}=0, c_{1, L}^{0}=0$ and $c_{2, L}^{0}=1$. Then formula (7) shows that $c_{1, \sigma}^{0}=c_{2, \sigma}^{0}=0$. Therefore, if $\kappa^{\star}$ is set to 0 , then $\mathbf{c}_{\mathscr{T}}^{0}$ is a steady solution to the 
scheme, which is not reasonable for the discretisation of the heat equation. The introduction of $\kappa^{\star}>0$ annihilates this spurious solution.

\section{Some pieces of numerical analysis}

Our first statement deals with positivity preservation, mass conservation, the preservation of the constraint $\sum_{i} c_{i}=1$, and with the existence of a solution to the nonlinear system (5)-(7).

Proposition 1. Given $\mathbf{c}_{\mathscr{T}}^{n-1} \in \mathscr{A}$, then there exists (at least) one approximate solution $\mathbf{c}_{\mathscr{T}}^{n}$ to the scheme such that $\mathbf{c}_{\mathscr{T}}^{n} \in \mathscr{A}$.

Proof. In order to carry out the proof, one first needs to replace $c_{i \sigma}^{n}$ (and $c_{j \sigma}^{n}$ ) by $\widetilde{c}_{i \sigma}^{n}=c_{i \sigma}^{n} / \max \left(1, \sum_{\ell} c_{\ell \sigma}^{n}\right)$ in (6). These two quantities will be shown later on to coincide as $\sum_{\ell} c_{\ell \sigma}^{n} \leq 1$ on all the internal edges $\sigma$.

As a first step to prove that $\mathbf{c}_{\mathscr{T}}^{n} \in \mathscr{A}$, let us prove by contradiction that $c_{i, K}^{n} \geq 0$ for all $K \in \mathscr{T}$ and all $i \in\{1, \ldots, I\}$. Assume that $\min _{L} c_{i, L}^{n}<0$ for some $i$, and let $K$ be the cell where $c_{i, K}^{n}<0$ is minimum among all $c_{i, L}^{n}, L \in \mathscr{T}$. Then (7) implies that $\widetilde{c}_{i, \sigma}^{n}=0$ for all $\sigma \in \mathscr{E}_{K}$ so that we deduce from (5)-(6) that

$$
\sum_{\sigma \in \mathscr{E}_{K}} a_{\sigma}\left[\kappa^{\star}\left(1-\sum_{j=1}^{I} \widetilde{c}_{j, \sigma}^{n}\right)\left(c_{i, K}^{n}-c_{i, L}^{n}\right)+\sum_{j=1}^{I} \kappa_{i j} \widetilde{c}_{j, \sigma}^{n}\left(c_{i, K}^{n}-c_{i, L}^{n}\right)\right]>0 .
$$

Using $\widetilde{c}_{j, \sigma}^{n} \geq 0, \sum_{j} \widetilde{c}_{j, \sigma}^{n} \leq 1$, and $c_{i, K}^{n} \leq c_{i, L}^{n}$ in previous inequality yields a contradiction, hence $c_{i, K}^{n} \geq 0$ for all $i$ and all $K$.

The fact that $\sum_{K \in \mathscr{T}} c_{i, K}^{n} m_{K}=\sum_{K \in \mathscr{T}} c_{i, K}^{n-1} m_{K}=\int_{\Omega} c_{i}^{0}$ follows directly from the conservativity of the fluxes (6). Finally, one readily checks from (6) that

$$
\sum_{i=1}^{I} J_{i, K \sigma}^{n}=\frac{\kappa^{\star}}{d_{\sigma}} \sum_{i=1}^{I}\left(c_{i, K}^{n}-c_{i, L}^{n}\right), \quad \forall \sigma=K \mid L \in \mathscr{E} .
$$

So summing (5) over $i$ shows that $s_{K}^{n}=\sum_{i=1}^{I} c_{i, K}^{n}$ satisfies the discrete heat equation

$$
\frac{s_{K}^{n}-s_{K}^{n-1}}{\tau_{n}} m_{K}+\kappa^{\star} \sum_{\sigma=K \mid L \in \mathscr{E}_{K}} a_{\sigma}\left(s_{K}^{n}-s_{L}^{n}\right)=0, \quad \forall K \in \mathscr{T} .
$$

Since $s_{K}^{n-1}=1$ for all $K \in \mathscr{T}$, so does $\left(s_{K}^{n}\right)_{K}$. Therefore, $\mathbf{c}_{\mathscr{T}}^{n}$ belongs to $\mathscr{A}$. Now, it follows from a simple convexity argument that the logarithmic mean $c_{i, \sigma}^{n}$ of $c_{i, K}^{n}$ and $c_{i, L}^{n}$ is smaller than the arithmetic mean, the sum of which over $i$ is equal to 1 . Therefore, $c_{i, \sigma}^{n}=\widetilde{c}_{i, \sigma}^{n}$. The existence proof then easily follows from a topological degree argument [8], see our companion paper [4] for details.

Refining the above proof, one can show that $c_{i, K}^{n}>0$ for all $K \in \mathscr{T}$ as soon as $\int_{\Omega} c_{i}^{0}>0$. This property is key for the proof of our next statement, and is rigorously 
established in [4]. Our second statement highlights the energy diminishing character of the scheme, which should be thought as a discrete counterpart of (2).

Proposition 2. Let $\mathbf{c}_{\mathscr{T}}^{n} \in \mathscr{A}$ be a solution to the scheme as in Proposition 1, then

$$
\mathfrak{E}\left(\mathbf{c}_{\mathscr{T}}^{n}\right) \leq \mathfrak{E}\left(\mathbf{c}_{\mathscr{T}}^{n-1}\right)
$$

Proof. Without loss of generality, we assume that $\int_{\Omega} c_{i}^{0}>0$ for all $i$ (otherwise $c_{i, K}^{n}=0$ for all $K \in \mathscr{T}$ thanks to Proposition 1). Since $c_{i, K}^{n}>0$, one can multiply (5) by $\log \left(c_{i, K}^{n}\right)$ and to sum over $K \in \mathscr{T}$ and $i \in\{1, \ldots, I\}$, which leads to

$$
A+B:=\sum_{i=0}^{I} \sum_{K \in \mathscr{T}} \frac{c_{i, K}^{n}-c_{i, K}^{n-1}}{\tau_{n}} \log \left(c_{i, K}^{n}\right) m_{K}+\sum_{i=0}^{I} \sum_{K \in \mathscr{T}} \sum_{\sigma=K \mid L \in \mathscr{E}_{K}} m_{\sigma} J_{i, K \sigma}^{n} \log \left(c_{i, K}^{n}\right)=0 .
$$

Thanks to the convexity of $c \mapsto c \log c-c$ and to mass conservation, one has

$$
A \geq \frac{1}{\tau_{n}} \sum_{i=0}^{I} \sum_{K \in \mathscr{T}}\left(c_{i, K}^{n} \log c_{i, K}^{n}-c_{i, K}^{n-1} \log c_{i, K}^{n-1}\right) m_{K}=\frac{\mathfrak{E}\left(\mathbf{c}_{\mathscr{T}}^{n}\right)-\mathfrak{E}\left(\mathbf{c}_{\mathscr{T}}^{n-1}\right)}{\tau_{n}} .
$$

The particular choice (7) for $c_{i, \sigma}^{n}$ allows us to rewrite

$J_{i, K \sigma}^{n}=\kappa^{\star} \frac{c_{i, K}^{n}-c_{i, L}^{n}}{d_{\sigma}}+\sum_{j \neq i} \widetilde{\kappa}_{i j} c_{i, \sigma}^{n} c_{j, \sigma}^{n}\left(\log \left(c_{i, K}^{n}\right)-\log \left(c_{j, K}^{n}\right)-\log \left(c_{i, L}^{n}\right)+\log \left(c_{j, L}^{n}\right)\right)$.

This implies that

$$
\begin{aligned}
B= & \kappa^{\star} \sum_{i=1}^{N} \sum_{\sigma=K \mid L \in \mathscr{E}} a_{\sigma}\left(c_{i, K}^{n}-c_{i, L}^{n}\right)\left(\log \left(c_{i, K}^{n}\right)-\log \left(c_{i, L}^{n}\right)\right) \\
& +\sum_{\{i, j\}} \sum_{\sigma=K \mid L \in \mathscr{E}} \widetilde{\kappa}_{i j} a_{\sigma} c_{i, \sigma}^{n} c_{j, \sigma}^{n}\left(\log \left(c_{i, K}^{n}\right)-\log \left(c_{j, K}^{n}\right)-\log \left(c_{i, L}^{n}\right)+\log \left(c_{j, L}^{n}\right)\right)^{2} .
\end{aligned}
$$

Since the logarithmic mean $c_{i, \sigma}^{n}$ of $c_{i, K}^{n}$ and $c_{i, L}^{n}$ is smaller than the arithmetic mean, there holds $\sum_{i} c_{i, \sigma}^{n} \leq 1$. As a consequence, one has

$$
\begin{aligned}
\sum_{i=1}^{N} & \sum_{\sigma=K \mid L \in \mathscr{E}} a_{\sigma}\left(c_{i, K}^{n}-c_{i, L}^{n}\right)\left(\log \left(c_{i, K}^{n}\right)-\log \left(c_{i, L}^{n}\right)\right) \\
& \geq \sum_{\{i, j\}} \sum_{\sigma=K \mid L \in \mathscr{E}} a_{\sigma} c_{i, \sigma}^{n} c_{j, \sigma}^{n}\left(\log \left(c_{i, K}^{n}\right)-\log \left(c_{j, K}^{n}\right)-\log \left(c_{i, L}^{n}\right)+\log \left(c_{j, L}^{n}\right)\right)^{2},
\end{aligned}
$$

which implies that

$$
B \geq \sum_{\{i, j\}} \sum_{\sigma} \kappa_{i j} a_{\sigma} c_{i, \sigma}^{n} c_{j, \sigma}^{n}\left(\log \left(c_{i, K}^{n}\right)-\log \left(c_{j, K}^{n}\right)-\log \left(c_{i, L}^{n}\right)+\log \left(c_{j, L}^{n}\right)\right)^{2} \geq 0 .
$$


This concludes the proof of Proposition 2.

A more involved study allows to show that under classical assumptions on nondegeneracy of the mesh regularity, then $\mathbf{c}_{\mathscr{T}, \tau}:(t, \mathbf{x}) \mapsto \sum_{n \geq 1} \mathbf{c}_{\mathscr{T}}^{n}(\mathbf{x}) \xi_{\left[t^{n-1}, t^{n]}\right.}(t)$ converges in $L_{\text {loc }}^{1}\left(\mathbb{R}_{+} \times \bar{\Omega}\right)$ towards a weak solution $\mathbf{c}$ to (1) provided $\kappa_{i j}>0$ for all $i, j$. The proof relies on the exploitation of the regularization coming from the dissipation (term $B$ in the proof of Proposition 2). We refer to [4] for the details of the convergence proof.

\section{Numerical results}

The numerical scheme has been implemented using MATLAB. The nonlinear system corresponding to the scheme is solved thanks to Newton method with stopping criterion $\left\|\mathbf{c}^{n, k+1}-\mathbf{c}^{n, k}\right\|_{\infty} \leq 10^{-12}$. The next iterate $\mathbf{c}^{n, k+1}$ is then "projected" on $\mathscr{A}$ by setting $\mathbf{c}^{n, k+1}=\max \left(\mathbf{c}^{n, k+1}, 10^{-10} \tau\right)$, and then $\mathbf{c}^{n, k+1}=\mathbf{c}^{n, k+1} /\left(\sum_{i=1}^{N} \mathbf{c}_{i}^{n, k+1}\right)$. For the first time step, we also make use of a continuation method based on the intermediate diffusion coefficients $\kappa_{i, j}^{\lambda}=\lambda \kappa_{i j}+(1-\lambda) \kappa^{\star}$ with $\lambda \in[0,1]$. The parameter $\lambda$ is originally set to 1 . If the Newton's method does not converge, we let $\lambda=\left(\lambda+\lambda_{\text {prev }}\right) / 2$ where $\lambda_{\text {prev }}$ is originally set to 0 . If the Newton's method converges, we let $\lambda_{\text {prev }}=\lambda$ and $\lambda=1$.

Our first test case is devoted to the convergence analysis of the scheme in a onedimensional setting $\Omega=(0,1)$. Two different initial conditions are considered: $\mathbf{c}_{s}^{0}$ is smooth with coordinates that vanish pointwise at the boundary of $\Omega$, whereas $\mathbf{c}_{r}^{0}$ is discontinuous with vanish coordinates on intervals of $\Omega$ :

$$
\begin{array}{lll}
c_{1, s}^{0}(x)=\frac{1}{4}+\frac{1}{4} \cos (\pi x), & c_{2, s}^{0}(x)=\frac{1}{4}+\frac{1}{4} \cos (\pi x), & c_{3, s}^{0}(x)=\frac{1}{2}-\frac{1}{2} \cos (\pi x), \\
c_{1, r}^{0}=1_{\left[\frac{3}{8}, \frac{5}{8}\right]}, & c_{2, r}^{0}=1_{\left(\frac{1}{8}, \frac{3}{8}\right)}+1_{\left(\frac{5}{8}, \frac{7}{8}\right)}, & c_{3, r}^{0}=1_{\left[0, \frac{1}{8}\right]}+1_{\left[\frac{7}{8}, 1\right]} .
\end{array}
$$

We also consider two diffusion matrices, one called regular with positive offdiagonal coefficients and an other called singular with a few null off-diagonal coefficients.

$$
K^{\mathrm{reg}}=\left(\begin{array}{ccc}
0 & 0.2 & 1 \\
0.2 & 0 & 0.1 \\
1 & 0.1 & 0
\end{array}\right) \quad K^{\text {sing }}=\left(\begin{array}{ccc}
0 & 0 & 1 \\
0 & 0 & 0.1 \\
1 & 0.1 & 0
\end{array}\right)
$$

For the convergence tests, we have let $\kappa^{\star}=0.1$ and the meshes are uniform discretisations of $[0,1]$ from $2^{5}$ cells to $2^{14}$ cells. Since we do not have an analytical solution at hand, the approximate solutions are compared to a reference solution computed on a grid made of $2^{15}$ cells. The final time is 0.25 , and the time discretisation is fixed with a time step of $2^{-18}$. Result are summarised in Figure 1. One notices that our scheme is second order accurate in the setting presented in this paper $\left(K=K^{\mathrm{reg}}\right)$, but only first order accurate when confronted to non-diffusive discontinuities. The 
origin of this lower order may lie in the difficulty to compute accurately the near zero concentrations in the neighbourhood of such discontinuities.

Our second test is two-dimensional. We choose $K^{\text {sing }}$ as the diffusion matrix, $\kappa^{\star}=0.1, \Omega=[0,22] \times[0,16], \tau=2^{-3}$ and a $2 \mathrm{D}$ initial condition $\mathbf{c}^{0}$ depicted in Figure 2. The corresponding steady state and long-time limit $\mathbf{c}^{\infty}$ does not depend on $\mathbf{x}$, i.e., $c_{i}^{\infty}(\mathbf{x})=\oint c_{i}^{0}(\mathbf{y}) \mathrm{d} \mathbf{y}$ for all $\mathbf{x} \in \Omega$. The time evolution of the relative energy $\mathfrak{E}(\mathbf{c})-\mathfrak{E}\left(\mathbf{c}^{\infty}\right)$ is plotted on Figure 3, showing exponential decay to the steady state even thought the diffusion matrix is singular. Snapshots showing the evolution of the concentration profiles are presented in Figure 4.

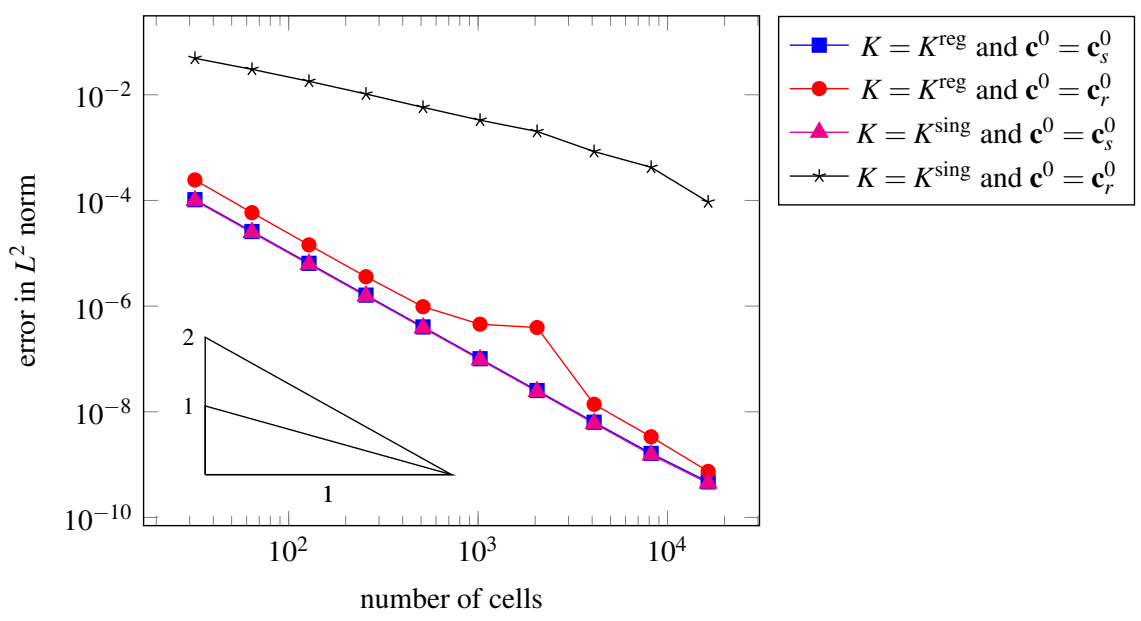

Fig. 1: Error with respect to the solution computed on the finest mesh for 1D settings

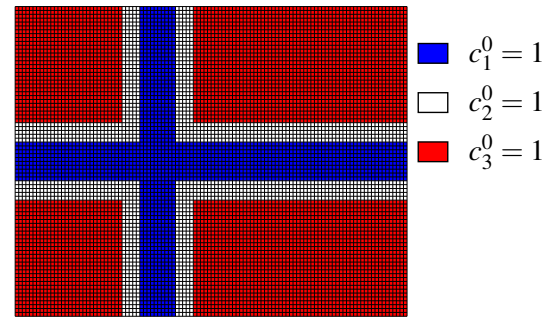

Fig. 2: Initial configuration $\mathbf{c}^{0}$

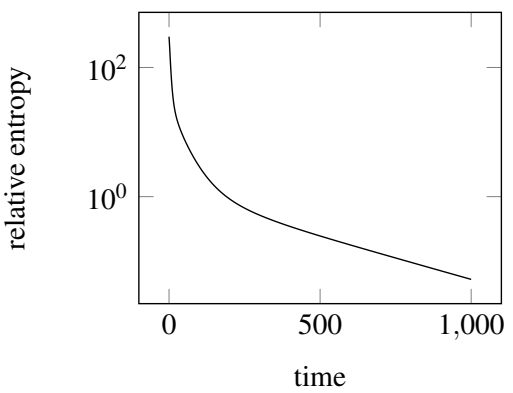

Fig. 3: $\mathfrak{E}(\mathbf{c})-\mathfrak{E}^{\infty}$ as a function of time. 


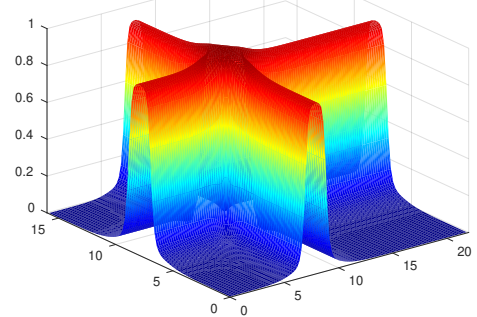

$c_{1}(t=2)$

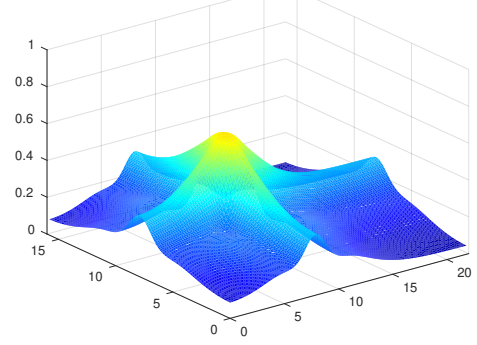

$c_{1}(t=10)$

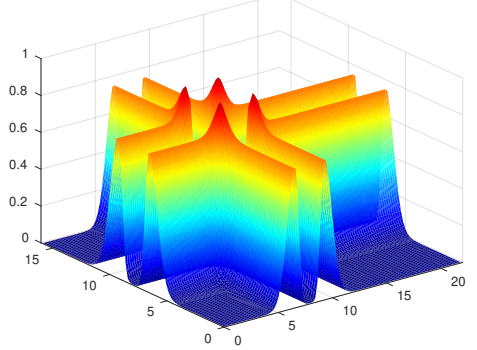

$c_{2}(t=2)$

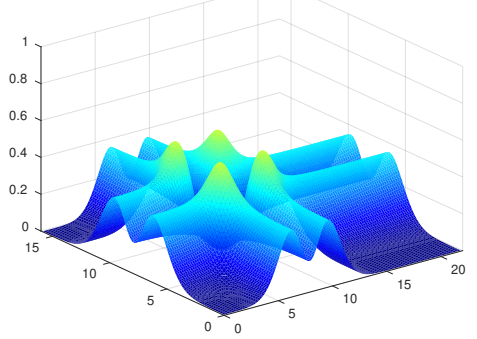

$c_{2}(t=10)$

Fig. 4: Concentrations $c_{1}$ and $c_{2}$ at times $t=2$ and $t=10\left(c_{3}\right.$ can be deduced from the relation $c_{1}+c_{2}+c_{3}=1$ ).

Acknowledgements The authors acknowledge the support of Labex CEMPI (ANR-11-LABX0007). Clément Cancès also aknowledges support from the COMODO project (ANR-19-CE460002), and he warmly thanks Virginie Ehrlacher and Laurent Monasse for stimulating discussions.

\section{References}

1. Bakhta, A., Ehrlacher, V.: Cross-diffusion systems with non-zero flux and moving boundary conditions. ESAIM Math. Model. Numer. Anal. 52(4), 1385-1415 (2018)

2. Berendsen, J., Burger, M., Ehrlacher, V., Pietschmann, J.F.: Uniqueness of strong solutions and weak-strong stability in a system of cross-diffusion equations. To appear in J. Evol. Equ.

3. Burger, M., Di Francesco, M., Pietschmann, J.F., Schlake, B.: Nonlinear cross-diffusion with size-exclusion. SIAM J. Math. Anal. 46(6), 2842-2871 (2010)

4. Cancès, C., Gaudeul, B.: A convergent entropy diminishing finite volume scheme for a crossdiffusion system (2020). HAL:hal-2460299

5. Eymard, R., Gallouët, T., Herbin, R.: Finite volume methods. Ciarlet, P. G. (ed.) et al., in Handbook of numerical analysis. North-Holland, Amsterdam, pp. 713-1020 (2000)

6. Jüngel, A.: The boundedness-by-entropy method for cross-diffusion systems. Nonlinearity 28(6), 1963-2001 (2015)

7. Jüngel, A.: Entropy methods for diffusive partial differential equations. SpringerBriefs in Mathematics. Springer, [Cham] (2016)

8. Leray, J., Schauder, J.: Topologie et équations fonctionnelles. Ann. Sci. École Norm. Sup. 51((3)), 45-78 (1934) 